\title{
Analysis of the Dynamic Landscape of Scientific Innovation in the Chip Domain
}

\author{
Jianqi Mao ${ }^{1,2}, \&$ Xiaxia Guo ${ }^{1}$ \\ ${ }^{1}$ Shandong Technology and Business University, Yantai, Shandong, China \\ ${ }^{2}$ Coal industry development and innovation research base, Yantai, Shandong, China \\ Correspondence: Xiaxia Guo, Shandong Technology and Business University, No.191, Laishan District, Yantai \\ City, Shandong Province, China.
}

Received: September 25, 2021

Accepted: October 20, 2021

Online Published: October 26, 2021

doi:10.5539/ibr.v14n12p1

URL: https://doi.org/10.5539/ibr.v14n12p1

\begin{abstract}
Chip is the core of the electronic information industry, and its current "neck" phenomenon is rooted in the failure to keep up with basic research. This study aims to provide a clear landscape of the dynamics of scientific innovation in the chip domain. Specifically, this study uses bibliometrics, social network analysis, and SPLC algorithm-based master path analysis to analyze spatial and temporal trends, collaboration characteristics, knowledge flows, and mainstream research paths through the global output of scientific paper in the field of the chip. The results show that the scientific output in this field has grown significantly, the network size is expanding, a few countries/regions and organizations are in the main position of chip science innovation, and the research themes on the mainstream research paths are concentrated in chip manufacturing. The study can provide lessons for researchers, policymakers, and research funders.
\end{abstract}

Keywords: chip, basic research, scientific innovation dynamics

\section{Introduction}

In the past three decades, the semiconductor industry has been one of the most important industries, which has been widely used in communications, consumer electronics, computers, and other fields, and has become the core upstream element of almost every part of the electronics industry. We are in an era of intelligence, digitalization, and informatization. In this trend, the semiconductor industry, with its pioneering, fundamental, and strategic nature, is an important indicator of a country's level of technological development and comprehensive national power (Lee, Swain, Gu, Lee, \& Yoon, 2021). Semiconductors are always the main battleground for the countries to carry out technology and trade wars. In the semiconductor industry, chip technology has always been a strategic high point for countries to compete fiercely.

In recent years, China has made great achievements in the field of science and technology and has become a very influential scientific and technological power. But in some key technology areas of the industry, there are still obvious shortcomings (Wang, 2020). The chip is the core foundation of the electronic information industry (Smith, Sonnenfeld, \& Pellow, 2006). As a key area of science and technology innovation, China's chip technology has long been restricted by others and the chip "neck" problem has become serious. To solve the "neck" problem, it is crucial to rely on science and technology innovation, which is an inevitable choice and a realistic requirement for the breakthrough of key core technologies in strategic industries. The existing research in the field of chips is mainly focused on the technical level(Manz, \& Eijkel, 2001), ignoring the basic research in this field. However, "the root of many 'neck' technology problems faced by China is that the basic theoretical research cannot keep up, and the source and the bottom are not clearly understood" (Xi, 2020). Wang(2020) also pointed out that the "neck" problem is mainly stuck in the less profound basic science and technology and basic research work. Therefore, it is necessary to conduct a systematic analysis of basic research, i.e., scientific innovation, in the field of the chip, which can help to understand a specific research area comprehensively. This study analyzes the dynamics of scientific innovation in the chip field at three levels: country/region, institution, and scientific paper aiming to provide a clear landscape of scientific innovation in the chip field, which helps to recognize the current status and development direction of the chip research field and provides valuable insights to optimize the layout of basic research in the chip field. 


\section{Literature Review}

\subsection{Research Related to the Chip Field}

The current academic research in the field of the chip is mainly focused on biochips and electronic chips. Biochips are commonly used in the medical field and can be widely used for disease diagnosis and treatment, drug screening, etc. In the field of biochips, existing studies mainly analyze the classification (Vo-Dinh, \& Cullum, 2000) and materials of biochips (Yamada, Shibata, Suzuki \& Citterio, 2017). In the field of electronic chips, existing studies mostly focus on the technology level from a patent perspective. For example, $\operatorname{Li}(2019)$ analyzed the overall situation of China's artificial intelligence chip industry in terms of patent output, patent value, and technology areas based on patent data. Cui and Zhang(2017) also using patent data in the chip field, pointed out the advantages and shortcomings of the domestic IC industry based on the analysis of the types and numbers of patents in this field, and discussed how Chinese enterprises would use patented technologies to improve their core competitiveness and take a place in the international arena. Other scholars have conducted some qualitative studies on the chip field. Wang, Tang, and $\mathrm{Xu}(2020)$ sorted out a group of major future chip technologies, analyzed the current development status, the maturity stage, and the prospect of these chip technologies, and made suggestions for the development of China's future chip technologies, intending to provide references for related research work in China. Zou(2020) achieved intelligent imaging quality evaluation by introducing AI chips and used an optical imaging processing system to solve the bottleneck problem of real-time and high quality faced by microwave imaging. Li, Chen, and Luo(2021) analyzed the development history of the atomic chip, introduced the basic principles of micro-potential wells and guided implementation of the atomic chip based on carrier wires, and focused on the full chain of key implementation technologies for the preparation technology, testing methods and integration of the atomic chip based on carrier wires. A few scholars applied the method of social network analysis to the research in the field of chips. Liu, Shi, Li and He (2020) used the analysis method of social network to establish a patent cooperation network with the region as the research object, analyzed the overall structure of the network and the characteristics of the nodes in stages, and studied the state of collaborative innovation in the field of chip technology in the Guangdong-Hong Kong-Macao Greater Bay Area.

\subsection{Research Related to Innovation Landscape Measurement}

Current research measures the innovation landscape mainly in terms of both science and technology. Because the scientific paper output is an important reflection to evaluate the innovation capacity and quality of scientific subjects (Guan, \& Liu, 2014). Technological innovation capacity is largely reflected in a country's patenting activities, which can be measured in terms of technical and economic values (Rivette, \& Kline, 2000). Therefore, innovation measurement and identification of a technology field using thesis data and patent data can accurately reflect the comprehensive strength of innovation in this field. Guan and Liu(2014) used scientometric (including bibliometrics and patent measurement) methods and social network analysis methods to measure scientific and technological innovation in the field of nano-energy and conducted an international comparative analysis based on technology innovation theory and complex network theory. Similarly, Mao, Sun, and Liu(2016) also measured the scientific innovation in general field of energy storage technology and each subfield using thesis data to provide a comprehensive analysis and presentation of the scientific research landscape of energy storage technology systems. In addition, the empirical studies of domestic and foreign scholars on measurement of technology innovation mainly focus on the construction of evaluation index system and the selection of evaluation methods(Boly, Morel, \& Camargo, 2014) Scholars mostly construct multi-level index systems based on two indicators of innovation input and innovation output. Cao, W.Zhang and Y.Zhang(2017) and Wang and Hou(2017) analyzed the dynamic evolution model of innovation in the high-tech industry from three perspectives of breadth, depth, and the life cycle.

\subsection{Comprehensive Review}

In summary, scholars have mostly conducted some qualitative studies on chip technology and focused on the technology level. Although a few scholars have conducted quantitative studies on the chip field, they are limited to the quantitative situation of the patent output of chip technology. In addition, there is a lack of an overall analysis of basic research in the chip field. Where is the scientific knowledge in the chip field mainly located, which organizations are the major players in chip research, how does the distribution of their innovations change over time, and what are the mainstream research paths in the field? Addressing these questions can help identify the current state and future direction of the chip field, and thus provide insights for optimizing the layout of basic research.

To achieve this goal, this study employs an integrated approach of bibliometrics, social network analysis, and 
main path analysis to map the comprehensive innovation landscape of the chip field at three levels: country/region, institution, and scientific paper, and to compare their landscapes dynamically by dividing them into time windows, with the expectation that this study will provide insights into the development and deployment of basic research in the chip field.

\section{Research Methods and Data Sources}

\subsection{Research Methodology and Framework}

Overall, this study tracks the scientific paper output in the field of the chip promptly by capturing the publication year of the papers. Next, the dynamics of scientific innovation in the field of chip research are specifically analyzed at three levels.

At the country/region level, this study counts scientific papers from different countries/regions, analyzes the number of papers output and world share of TOP10 countries/regions, and introduces the AI index as an indicator to compare the relative effort levels of high-producing countries/regions. It has been shown that scientific collaboration can lead to a substantial improvement in the quality of innovation (Iino, Inoue, Saito, \& Todo, 2021), most likely because various knowledge is combined in the collaboration and the impact of international collaboration is much greater than the impact of domestic collaboration because foreign collaborators have knowledge that is not available domestically. Therefore, based on the international collaboration thesis, this study also maps the network of collaborative relationships between countries/regions that reflect knowledge flows.

At the organizational level, this study examines the organizations in the world that are mainly involved in basic research on chips. In addition, the heterogeneity of knowledge among organizations also has an impact on the quality of innovation, so this study maps the collaboration networks among TOP50 organizations as a way to understand the structure and characteristics of inter-organizational knowledge flows.

At the dissertation level, this study focuses on exploring the mainstream research paths in the field of chip-based research and which countries/regions occupy important positions in the mainstream research paths. The mainstream research paths in the field of chip research are realized based on citation networks. First, a citation network of papers with references pointing to the target literature is constructed, and then the current mainstream research paths in the field of chip research are identified based on the main path analysis, a process that reflects the diffusion and flow of knowledge. This citation-based approach was first proposed by Hummon and Dereian(1989), who introduced two path search algorithms, SPLC and SPNP, which were later extended by Verspagen(2007). Since the SPLC algorithm focuses on both the relationship between source to sink literature and the contribution of intermediate literature, the citation network in this study uses the SPLC algorithm to calculate the traversal weights. Existing studies have introduced several algorithms for identifying primary research trajectories, including local master paths, global master paths, and critical path master paths(Liu, \& Lu, 2012). However, both local and global main paths suffer from a limitation that not all connections with the highest SPLC values can be identified by these two algorithms. The key-route main path can overcome this limitation(Liu, \& Lu, 2012). So the key-route algorithm is used in this study to reveal the main path problem in chip research. The process of main path analysis is implemented by pajek software.

\subsection{Data Sources}

The data of the papers used in this study was obtained from the SCI-E and SSCI literature databases of Web of Science. The complex word search strategy was selected, referring to the expressions of Liu, Yan, Cheng and Ye(2018), Liu(2021), and Chen \& Liu(2020) for the proper nouns in the field of the chip, and the search formula was constructed as shown in Table 1. The search language was set to English, the literature type was set to Article, the period time was all years, and the search date was June 6, 2021. A total of 41174 scientific papers was obtained.

Table 1. Search terms for papers in the field of the chip

\begin{tabular}{l} 
Search terms \\
\hline \#1 TS=(("Integrated Circuit" OR Semiconductor) AND chip*) \\
\#2 TS=((IC OR "Integrated Circuit" OR Semiconductor) AND ("system* design" or "RTL code* design" or "RTL \\
simulat*" or "logic synthesis" or "static timing analysis" or "functional verificat*" or "Design For Test" or "Test* \\
design" or "Physical layout" or (Extract* and "parasitic parameter*") or ("Physical verificat"” and layout))) \\
\#3 TS=((IC OR "Integrated Circuit" OR Semiconductor) AND ("device manufact*" or "Plasma clean*" OR \\
"Megasonic clean*" OR "Laser clean*" OR "X-ray Lithograph*" OR "Focused Icon Beam Lithograph*" OR \\
"Extreme Ultraviolet Lithograph*" OR "Nanoimprint Lithograph*" OR "Electron Projection Lithograph*" OR \\
"Wet Chemical Etch*" OR "Plasma Etch*" OR "Reactive Ion Etch*" OR "Inductively Coupled Plasma Etch*" \\
\hline
\end{tabular}




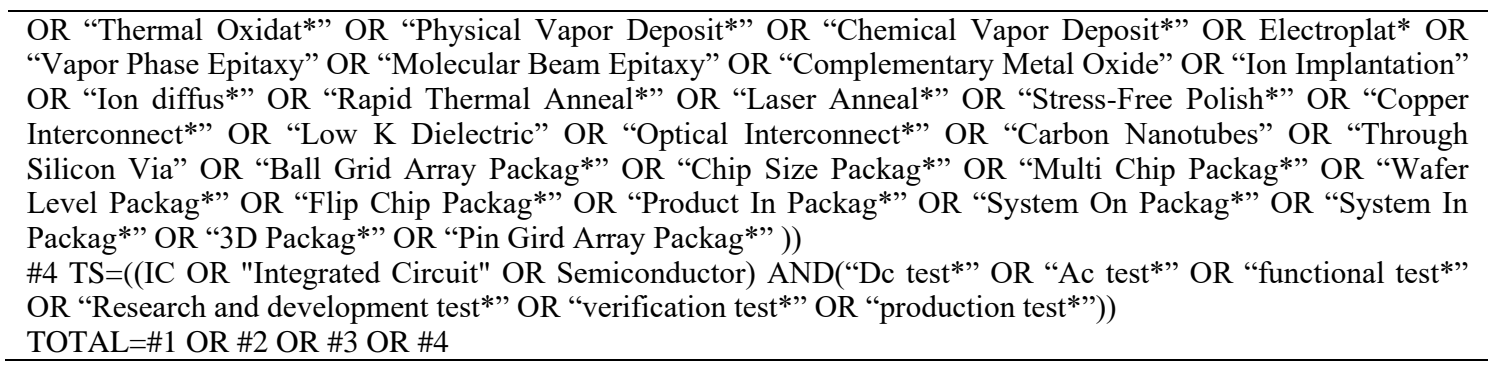

\section{Results}

This study first shows the overall trend of global scientific publications in the field of chips, as shown in Figure 1. A few scholars initially dabbled in this research area before the 1990s, but the research output was relatively small, with only 143 scientific publications. The first growth in scientific publications in the field of chips was achieved in 1991, and steady growth was achieved in the following almost 20 years, with an average annual growth rate of 12\%, with 1997 1998 and 2008 2009 to achieve significant growth of up to 58\% and 28\%. But between 2009 2012, there was a downward trend in scientific output in the field, and after 2012 began to grow slowly with a growth rate of only 5\%, with a relative increase in the growth rate of 15\% from 2019 2020. Throughout the period time, although the scientific output in the field of chips has gone through an unstable phase of development, it has generally shown a growth pattern. Due to the small amount of scientific literature in the field before 1991, it is difficult to show a clear scientific landscape. In this study, scientific papers during the 30 years from 1991 2020 are selected and divided into three-time windows according to a 10-year interval to depict the dynamic landscape of scientific research in the chip field.

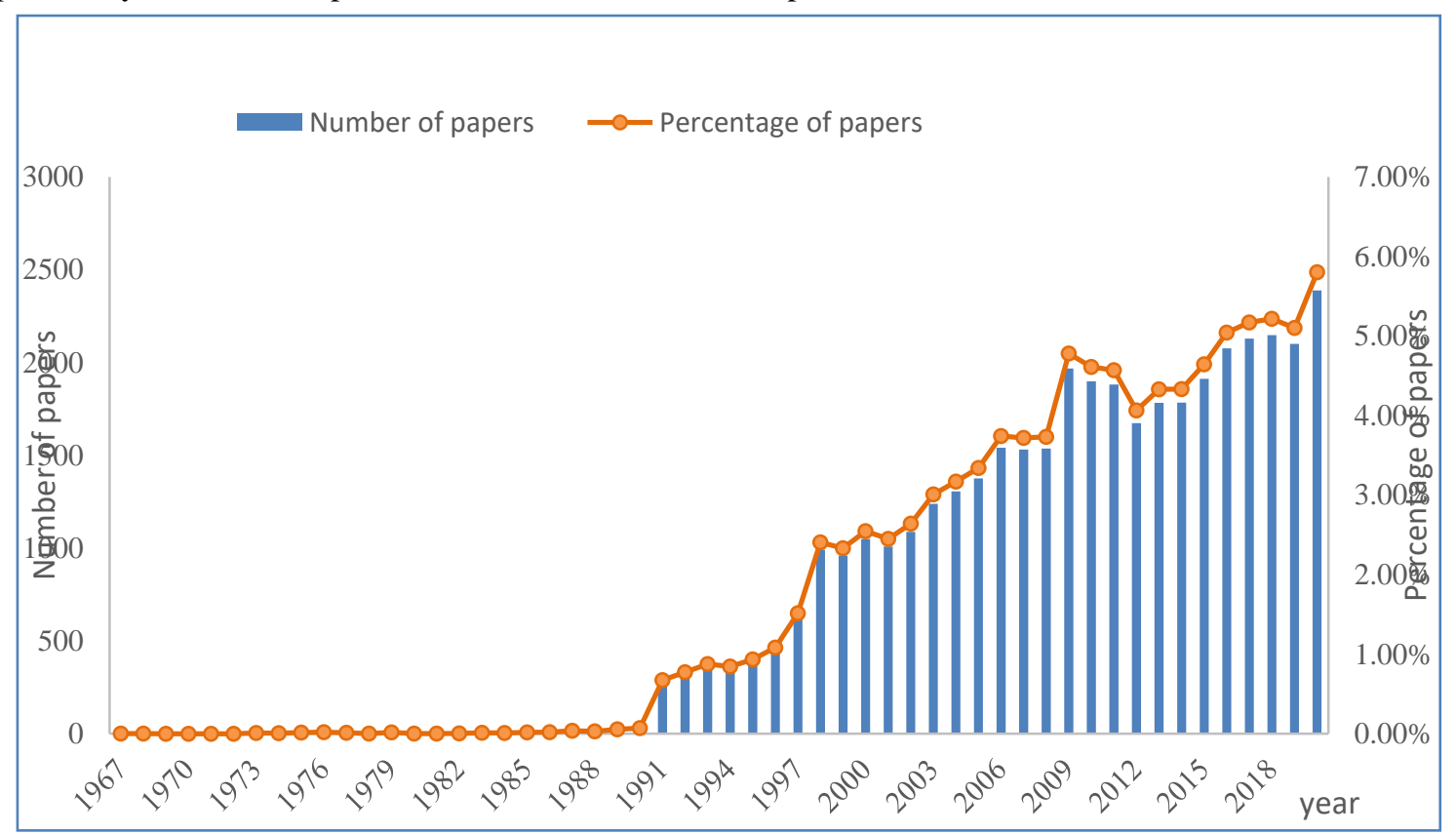

Figure 1. The yearly output of scientific papers published in the field of the chip

\subsection{Country/Region Level}

\subsubsection{High-Producing and High-Quality Countries/Regions}

Figure 2. gives the scientific paper output and world share in the field of chips for the period 1991 2020 for the TOP 10 countries/regions, which contributed $94.19 \%$ of the global scientific literature in the field of the chip. As shown in the figure, the U.S. scientific research output in the field of chips reaches nearly one-third (28.10\%) of the world's total output and is the most active player in the field of chips worldwide, China ranks second in the world with $14.56 \%$ of the world's papers in the field of chips, and China and the U.S. are the most prominent contributors in this field, followed by Japan (12.42\%), Germany $(8.98 \%)$, South Korea $(7.99 \%)$, Taiwan, $(6.32 \%)$, and France $(5.15 \%)$, these countries/regions are in the middle level of scientific output in the field of 
chips, and the remaining countries/regions have a weak level of output, none of which is higher than 5\%.

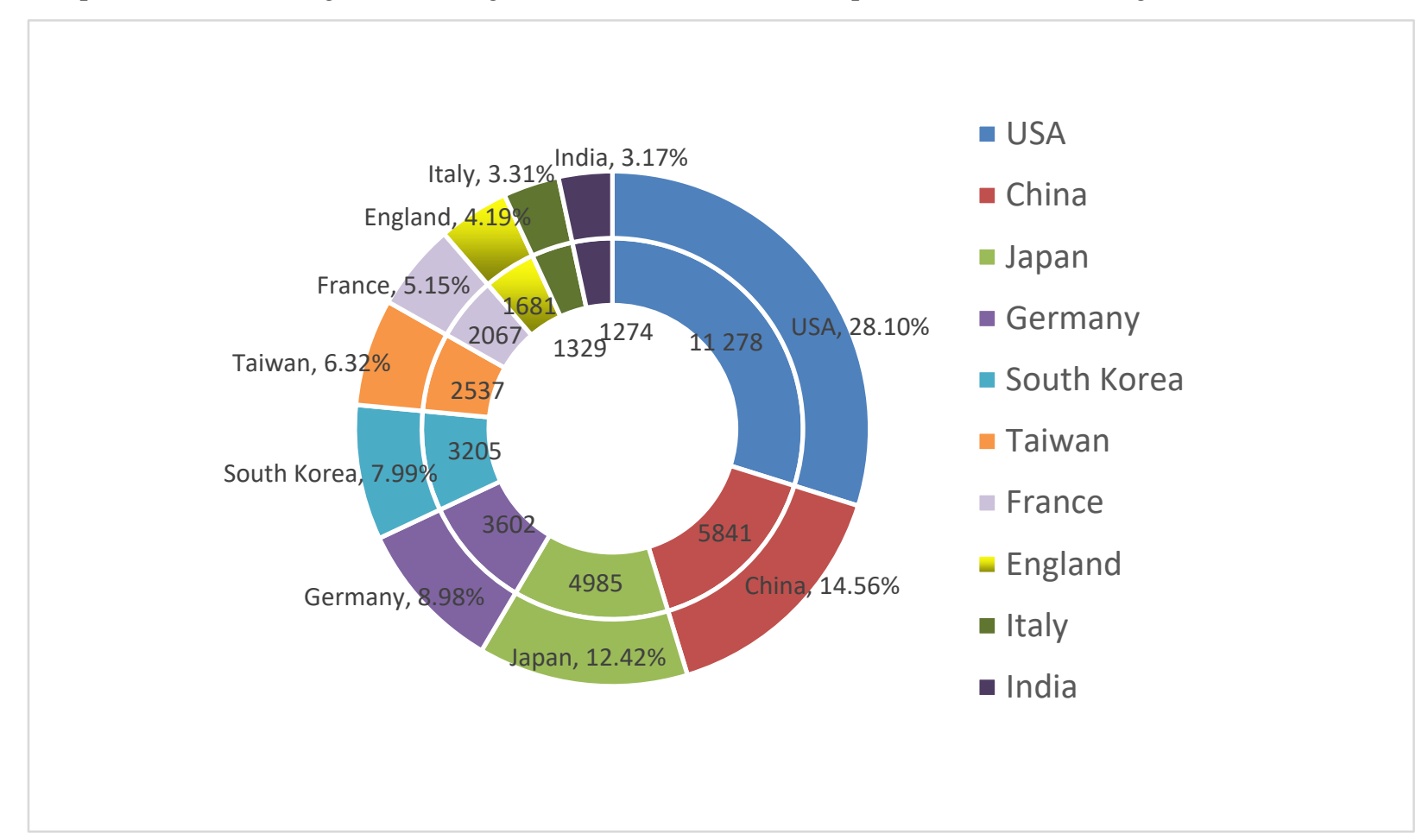

Figure 2. Number and world share of scientific papers in the field of the chip in the top 10 countries/regions

Figure 3 shows the evolution of the number of scientific outputs in the chip field over time, reflecting the dynamics of the innovation outputs of the TOP 10 countries/regions in this field. As shown in the figure, although the U.S. occupied the highest position at the beginning and has been leading in the number of outputs, its scientific output in the chip field has generally shown a decreasing trend. China's output level was lower than that of Japan, Germany, and the U.K. in the early stage, but showed a steady growth trend since 2003 and surpassed the U.S. as the world's top chip paper producing country in 2017. Except for Japan, which experienced an unstable development phase during the period, the output curves of all other countries are relatively flat.

To attenuate the effect of papers' output size, this study introduces the Activity Index (AI) index (Frame, 1977) to measure the relative research effort of each country/region. The index is calculated by the formula:

$$
A I_{i}^{t}=\frac{\left(P_{i}^{t} / \sum P\right)}{\left(T P^{t} / \sum T P\right)}
$$

Where $A I_{i}^{t}$ refers to the $\mathrm{AI}$ index of country $\mathrm{i}$ in year $\mathrm{t}, P_{i}^{t}$ represents the number of papers output in the field of chips in country $\mathrm{i}$ in year $\mathrm{t}, \sum P$ represents the total number of papers output in chips in that country in the research period (1991 2020 in this paper), ${ }^{T P}$ represents the total number of papers output in chips in the world in year $\mathrm{t}, T P^{t}$ represents the total number of papers output in chips in the research period (1991 2020 in this paper). When the AI value is equal to 1, it indicates that the research effort of the country/region in the field of the chip is equal to the world average; when the AI value is greater than 1, it indicates that the research effort of the country/region in the field of the chip is higher than the world average; when the AI value is less than 1, it indicates that the research effort of the country/region in the field of the chip is less than the world average.

Table 2 shows the AI values of the TOP 10 countries/regions. From the table, we can see that the relative effort made by the U.S. in the field of chips is gradually decreasing, but in general, it is still in the leading position, and its 30-year average is still higher than the world average, and the relative effort of China is increasing year by 
year, and it is higher than the world average after 2011. In addition to the United States, the 30-year average is higher than the world average in Japan and the United Kingdom, while the relative effort of other countries is lower than the world average.

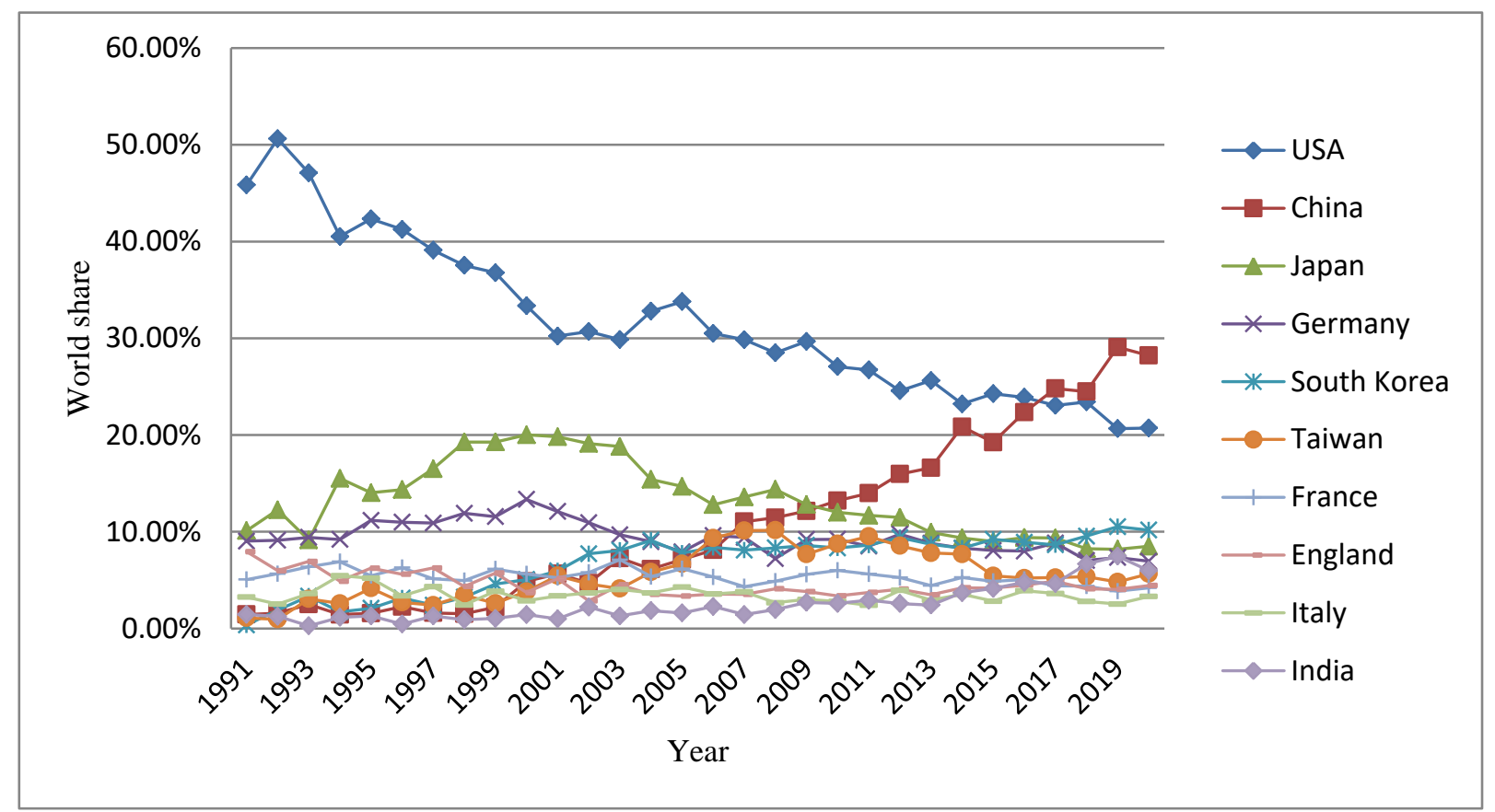

Figure 3. Revolution of the number of scientific papers produced by the TOP 10 countries/regions in the field of the chip 1991 2020

Table 2. Top 10 chip field country/region AI values

\begin{tabular}{ccccccccccc}
\hline Year & USA & China & Japan & Germany & $\begin{array}{c}\text { South } \\
\text { Korea }\end{array}$ & Taiwan & France & England & Italy & India \\
\hline $1991-1995$ & 1.52 & 0.11 & 0.93 & 1.01 & 0.22 & 0.35 & 1.08 & 1.44 & 1.14 & 0.32 \\
$1996-2000$ & 1.26 & 0.16 & 1.36 & 1.23 & 0.43 & 0.45 & 1.03 & 1.15 & 0.96 & 0.30 \\
$2001-2005$ & 1.05 & 0.40 & 1.33 & 1.04 & 0.91 & 0.79 & 1.09 & 0.87 & 1.08 & 0.47 \\
$2006-2010$ & 0.98 & 0.72 & 0.99 & 0.94 & 0.98 & 1.37 & 0.95 & 0.83 & 0.90 & 0.65 \\
$2011-2015$ & 0.83 & 1.12 & 0.78 & 0.91 & 1.04 & 1.16 & 0.93 & 0.88 & 0.89 & 0.93 \\
$2016-2020$ & 0.75 & 1.67 & 0.66 & 0.80 & 1.13 & 0.78 & 0.80 & 0.99 & 0.92 & 1.75 \\
Average value & 1.07 & 0.70 & 1.01 & 0.99 & 0.79 & 0.82 & 0.98 & 1.03 & 0.98 & 0.74 \\
\hline
\end{tabular}

\subsubsection{Cross-National/Regional Scientific Cooperation}

In this study, we constructed three periods of national/regional scientific cooperation networks based on the cooperation relationship between countries/regions from 1991 2000, 2001 2010, and 2011 2020 to analyze the dynamic evolution of the scientific cooperation network in the chip field, extracted the maximum connectivity diagram as shown in Figure 4 6, the nodes represent a country/region, the larger the node area indicates the more collaborators, the thicker the edge indicates the higher the frequency of its cooperation, and calculated the index reflecting the characteristics of the scientific cooperation network as shown in Table 3.

It can be visually seen from Figures 4 6 that the number of nodes and edges in the three phases of the network has increased significantly and the network has expanded steadily, as confirmed by the data in Table 3. Only 75 countries/regions in the network of scientific cooperation in 1991 2020, and the size of the network has reached 110 countries/regions in the 2011 2020 phase, which indicates that more and more countries/regions are joining the cooperation boom. The number of edges is even more significantly increased relative to the nodes, from 335 in 1991 2000 to 1164 in 2011 2020, i.e., more and more countries/regions are creating new partnerships and the partnership continues to expand. Table 3 shows that the average degree centrality of scientific networks in the 1991 2000 phase is 8.9, i.e., on average, each country/region has 8.9 other countries/regions with which to establish cooperation, and it develops to 21.2 in the third phase, and the worldwide cooperation is getting closer. 
The network densities of the three stages are $12 \%, 19 \%$, and $19 \%$ respectively, and the density has a tendency to increase year by year, but it is not too dense yet, and there is room for development.

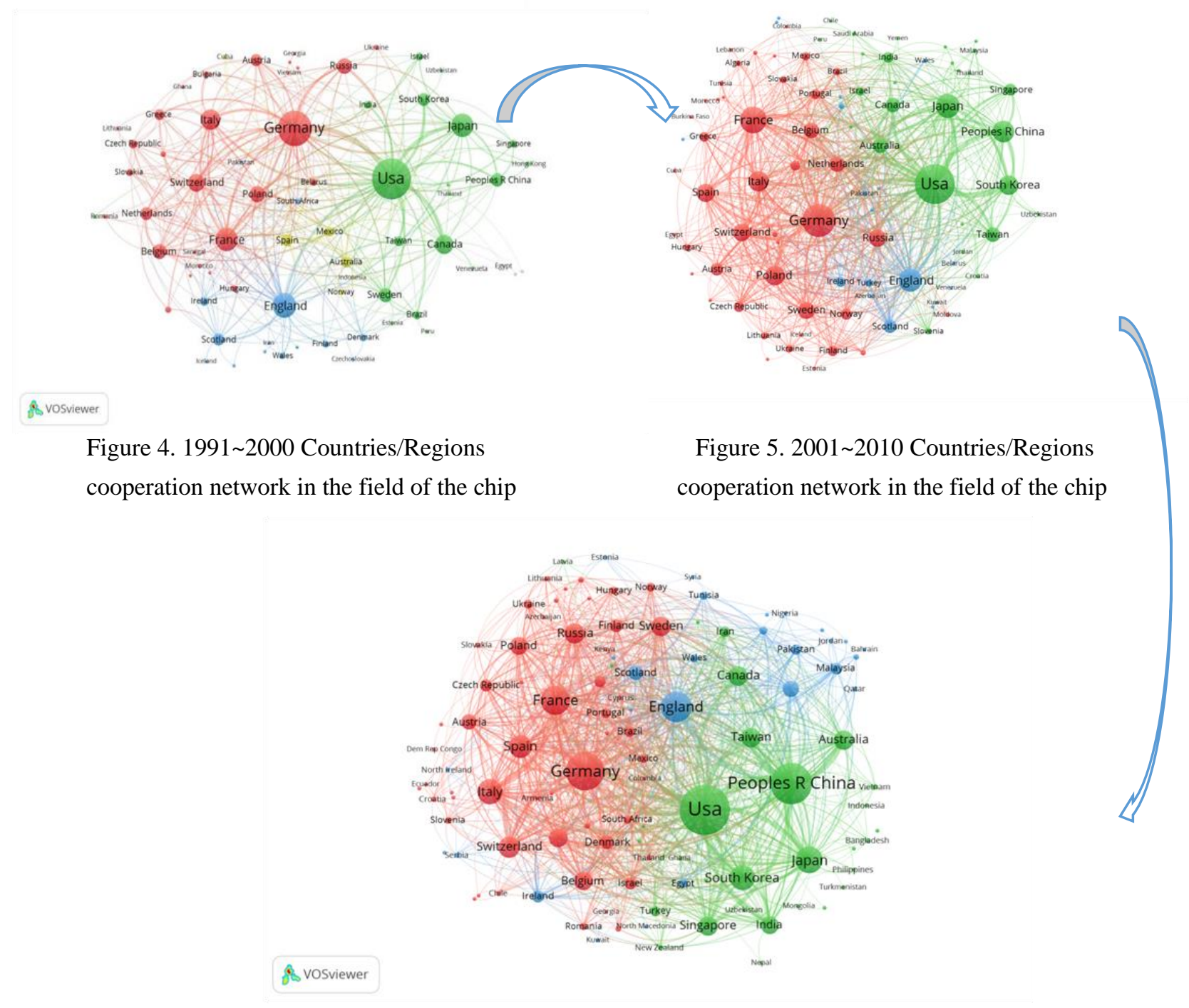

Figure 6. 2011 2020 Countries/Regions cooperation network in the field of the chip

According to Figure 4 6, all three phases of the network have formed a cooperation group with Germany, the United States, and the United Kingdom as the core respectively. Those who cooperate more closely with mainland China are the United States, Japan, Canada, South Korea and, Taiwan. Those who cooperate more closely with Germany are France, Spain, Italy, and other Western European countries. The United States and Germany have been at the core of the network and are the countries with stronger intensity in the world. China was initially located at the edge of the network, with smaller nodes and lower cooperation, and over time, China has gradually moved to the core of the network, and by the third stage has been comparable to the United States and Germany.

Table 3. Characteristics of national/regional scientific cooperation networks in the field of the chip

\begin{tabular}{cccc}
\hline Network Characteristics & $1991 \sim 2000$ & $2001 \sim 2010$ & $2011 \sim 2020$ \\
\hline Number of nodes & 75 & 90 & 110 \\
Number of links & 335 & 763 & 1164 \\
Average degree & 8.9 & 17.0 & 21.2 \\
Density & $12 \%$ & $19 \%$ & $19 \%$ \\
Average shortest path & 2.3 & 2.0 & 1.97 \\
Diameter & 5 & 4 & 4 \\
\hline
\end{tabular}




\subsection{Institutional Level}

\subsubsection{Highly Productive Organizations}

Since the same organizational institution may have different forms of names, to ensure the accuracy of the data, this study standardized the organizational institutions by first using Excel to automatically deduplicate them, then using Sci2 Tool's function of detecting duplicate points, and finally cleaning them manually. Table 4 shows the TOP 20 organizational bodies of scientific research outputs in the field of the chip. It also shows the nationality, type, and output information of institution's location. As can be seen from the table, $30 \%$ of the TOP 20 institutions in the field of chip scientific output are from the U.S. It is noteworthy that Taiwan is in second place, accounting for $20 \%$, with the U.S. and Taiwan, occupying half of the share. This is followed by countries such as France, Singapore and Italy. In addition, most of the TOP20 institutions are universities, with a proportion of $70 \%$, which indicates that the main innovators in the field of chip research are universities, followed by research institutes, and several major research institutes in the world are also the main players in basic research in the chip field. The only company present in the TOP20 in the chip field is IBM in the United States and is ranked fifth, with an output of 489 articles. It can be seen that two Chinese institutions are among the top in the world, namely the Chinese Academy of Sciences and Tsinghua University, which reveals that these two institutions are more active in the field of chip research.

Table 4. Top 20 institutions of scientific innovation output in the field of the chip

\begin{tabular}{cccc}
\hline Institution Name & Country/region & Institution Type & $\begin{array}{c}\text { Scientific Research } \\
\text { Output }\end{array}$ \\
\hline University of California & USA & University & 1526 \\
Chinese Academy of Sciences & China & Scientific Research Institutes & 1427 \\
National Chiao Tung University & Taiwan & University & 517 \\
Tokyo University & Japan & University & 514 \\
IBM & USA & Enterprise & 489 \\
CNRS & France & Scientific Research Institutes & 467 \\
Tohoku University & Japan & University & 435 \\
Russian Academy of Sciences & Russia & Scientific Research Institutes & 408 \\
National Tsing Hua University & Taiwan & University & 397 \\
European Microelectronics Research & Belgium & Scientific Research Institutes & 392 \\
Center & University & 391 \\
Nanyang Technological University & Singapore & University & 391 \\
MIT & USA & University & 380 \\
National Cheng Kung University & Taiwan & University & 376 \\
Taiwan University & Taiwan & University & 364 \\
Osaka University & Japan & University & 350 \\
Georgia Institute of Technology & USA & University & 343 \\
University of Illinois & USA & University & 343 \\
Stanford University & USA & Italy & Scientific Research Institutes \\
CNR & China & University & 331 \\
Tsinghua University & & & 329 \\
\hline
\end{tabular}

\subsubsection{Cross-institutional Scientific Collaboration}

In this study, we first extracted the TOP50 organizations of output in the chip field in three-time windows of 1991 2000, 2001 2010 and 2011 2020. And then constructed the cross-organizational cooperation network based on the cooperation relationship between them and analyzed its dynamic evolution. The cross-organizational cooperation network diagram is shown in Figure 7 9. The nodes represent an organization, the larger the node area indicates more collaborators, the thicker the edge indicates the higher the frequency of collaboration. The indicators reflecting the characteristics of the institutional scientific collaboration network are calculated, as shown in Table 5.

It can be visually seen from Figures 7 9 that the collaboration network of organizations and institutions in the chip field expands significantly, and combined with Table 5, the number of edges, i.e., the frequency of collaboration of collaborators, grows from 166 in the first stage (1991 2000) to 493 in the third stage (2011 2020), which indicates that innovators are breaking their limitations and implementing cross-organizational knowledge search step by step, and also reflects the further increase of knowledge flow between organizations. The organizational collaboration network in the first stage (1991 2000) shows a loose structure with a low density of $14 \%$, and there is still much room for improvement. While the network in the third stage (2011 2020) shows a tightly connected topology with an increase in density to $40 \%$, and the average number of collaborations among innovators (reflected in the average degree centrality in Table 5) increases 
significantly.

In this study, the three phases of the organization-institution cooperation network were matched by country, and it was found that the U.S. was always in a dominant position. The proportion of institutions affiliated with the U.S. in the first phase of the network was as high as $46 \%$, followed by Japan, which occupied $22 \%$. While in the first phase there was only one Chinese institution, the Chinese Academy of Sciences, which reflected the leading position of the Chinese Academy of Sciences in the domestic chip basic research field. The proportion of U.S. and Japanese institutions declined in the second stage, at 34\% and 16\% respectively, with three new Chinese innovators entering, namely Tsinghua University, Peking University and Nanjing University, with the University of California occupying a central position in the network in the first two stages. By the third phase, China's new entrant innovators increased significantly, with a $26 \%$ share, surpassing the United States as the top-ranked country in the third phase. And the nodes were significantly larger, especially the Chinese Academy of Sciences and the University of Chinese Academy of Sciences, which are world leaders. In addition, in terms of geography, all three phases of the collaboration network have formed distinct regional clusters, i.e., U.S. institutions prefer to collaborate with their own countries or neighboring countries such as the UK, while China's collaboration is dominated by domestic cooperation. In terms of cooperation mode, universities are the most numerous in the entrepreneur -university-scientific research institutes cooperation, followed by scientific research institutes, and enterprises account for a relatively low proportion, forming an obvious university-scientific research institutes cooperation, with a low degree of cooperation involving enterprises.

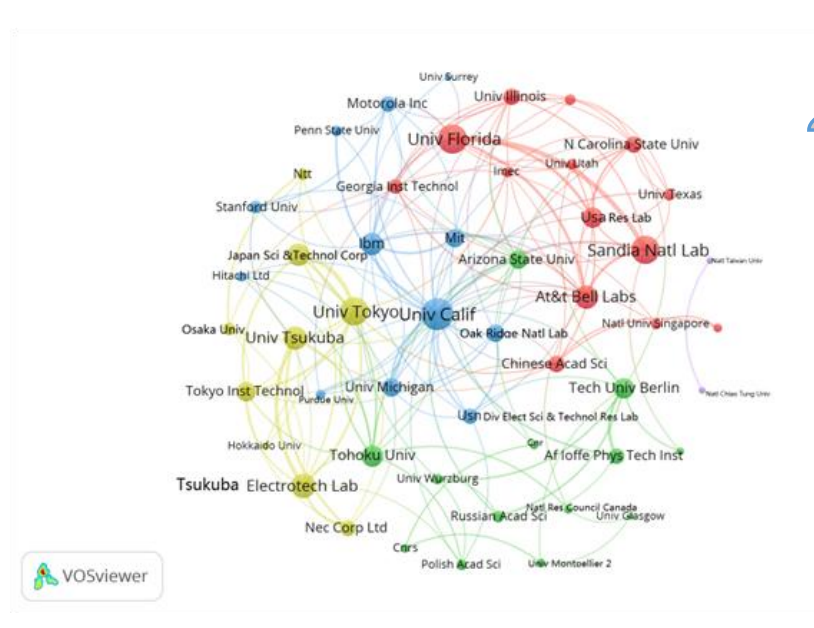

Figure 7. 1991 2000 Organization cooperation network in the field of the chip

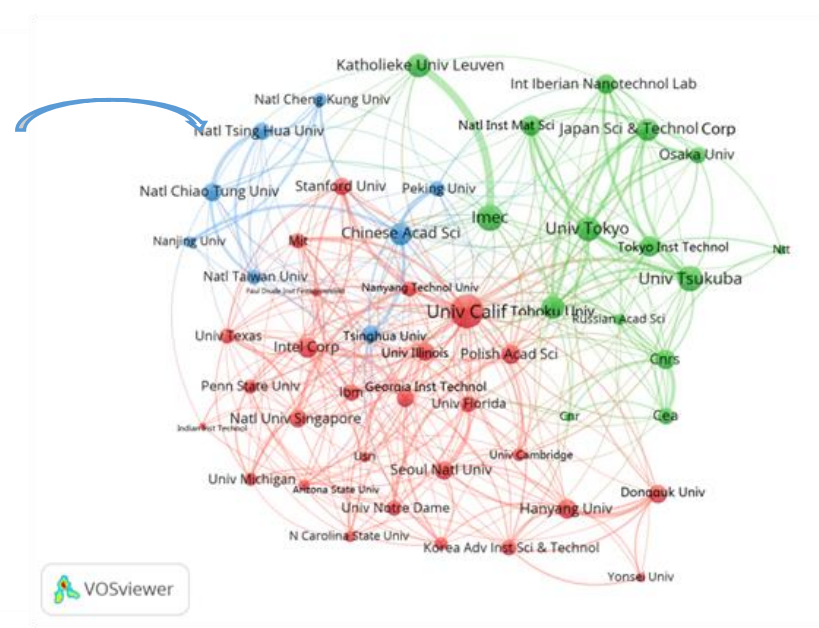

Figure 8. 2001 2010 Organization cooperation network in the field of the chip

\section{fis vosviewer}

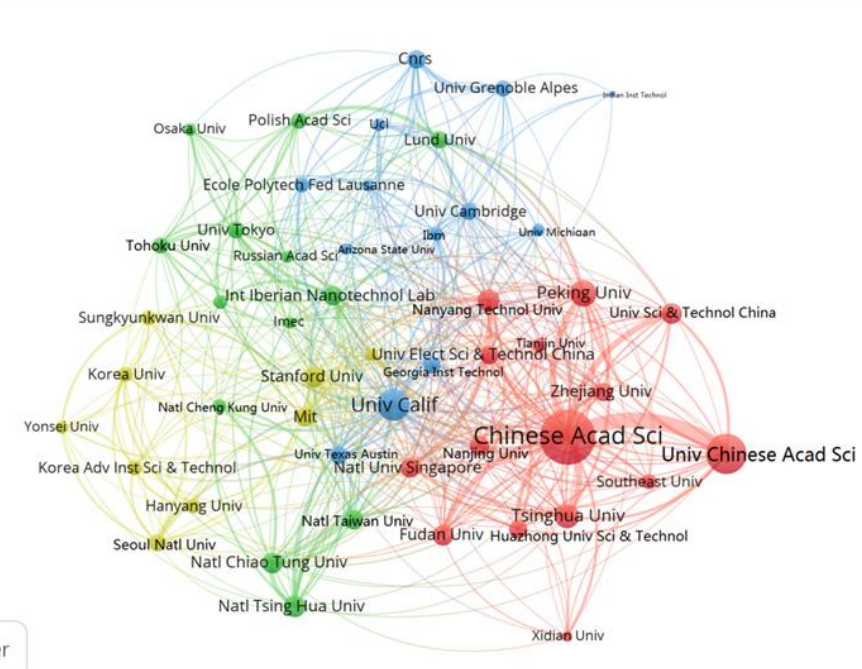

Figure 9. 2011 2020 Organization cooperation network in the field of the chip 
Table 5. Characteristics of cross-institutional scientific collaboration networks in the field of the chip

\begin{tabular}{cccc}
\hline Network Characteristics & $1991 \sim 2000$ & $2001 \sim 2010$ & $2011 \sim 2020$ \\
\hline Number of nodes & 166 & 344 & 493 \\
Number of links & 6.64 & 13.50 & 19.72 \\
Average degree & $14 \%$ & $27 \%$ & $40 \%$ \\
Density & 2.3 & 1.8 & 1.6 \\
Average shortest path & 5 & 4 & 3 \\
\hline
\end{tabular}

\subsection{Scientific Paper Level}

This study is based on paper citation network and master path analysis, using Pajek software to map the mainstream research paths in the chip field for three time periods 1991 2000,2001 2010,2011 2020. The method of master path analysis can simplify a complex research field into one or more master trajectories or master paths consisting of some key nodes and their connections, which reflects the flow and diffusion of knowledge in the chip field. In setting the threshold value for master path analysis, this study first uses the global standard algorithm to get the number of documents contained in the standard global master path and then sets the threshold value in the key-route master path algorithm based on this value. After trying, it is found that the master path shows a relatively obvious divergence-convergence pattern when the threshold values are 82 86, 58 62, and 60 64. Therefore, we set the threshold values according to the above values and generate the main research trajectory consisting of key nodes and arrows as shown in Figure 10 12, where the nodes represent a scientific paper and the arcs refer to the links between papers, indicating the flow of knowledge between papers.

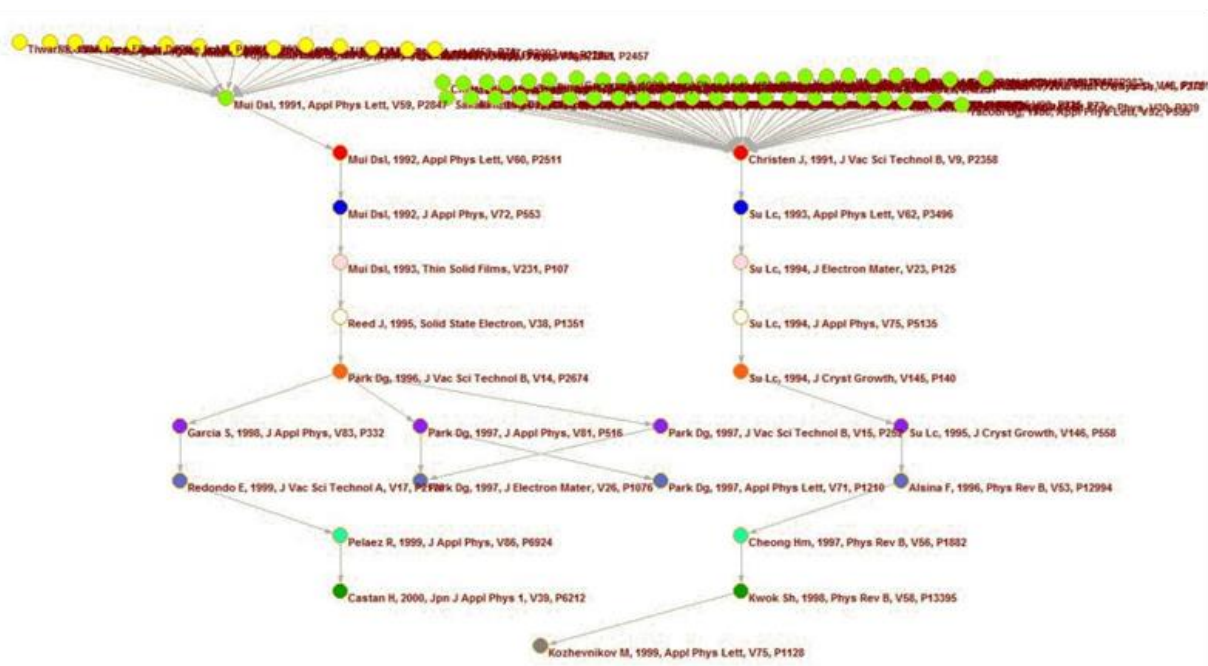

Figure 10. 1991 2000 Mainstream research paths in the field of the chip

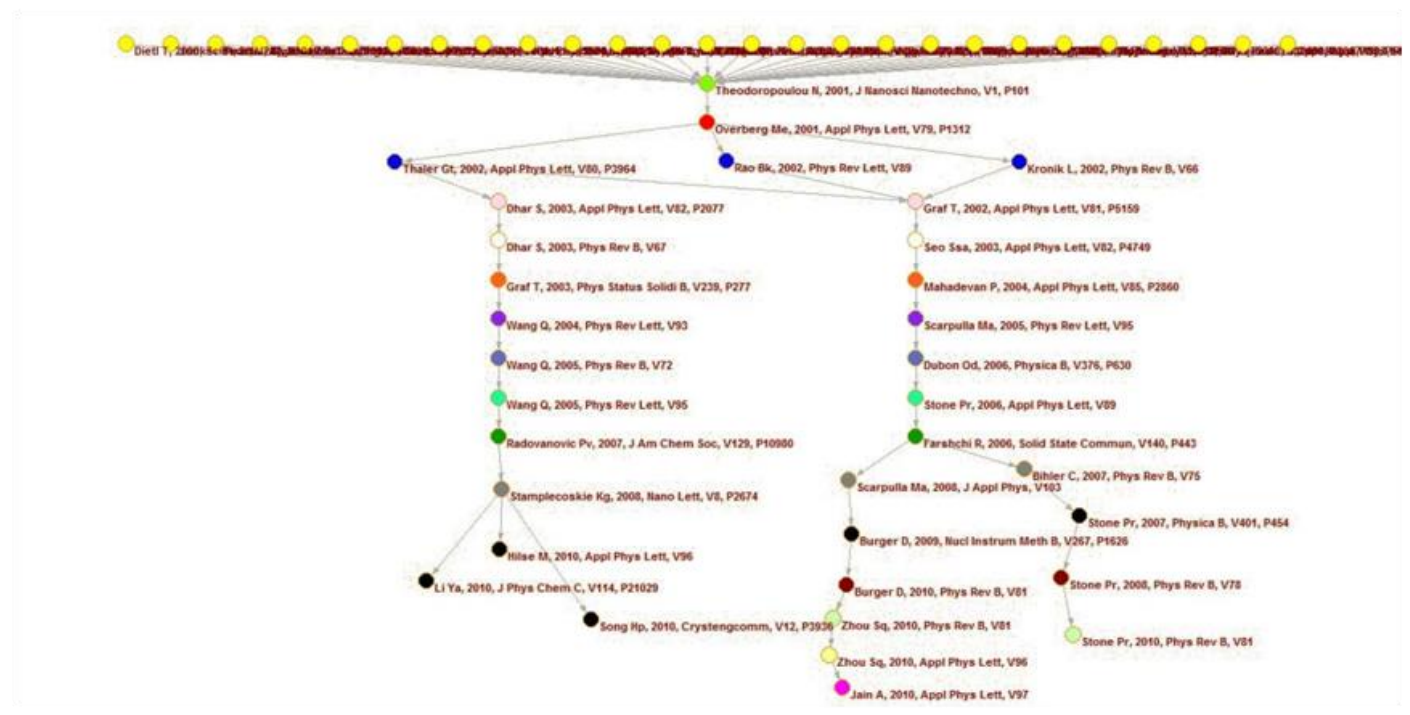

Figure 11. 2001 2010 Mainstream research paths in the field of the chip 


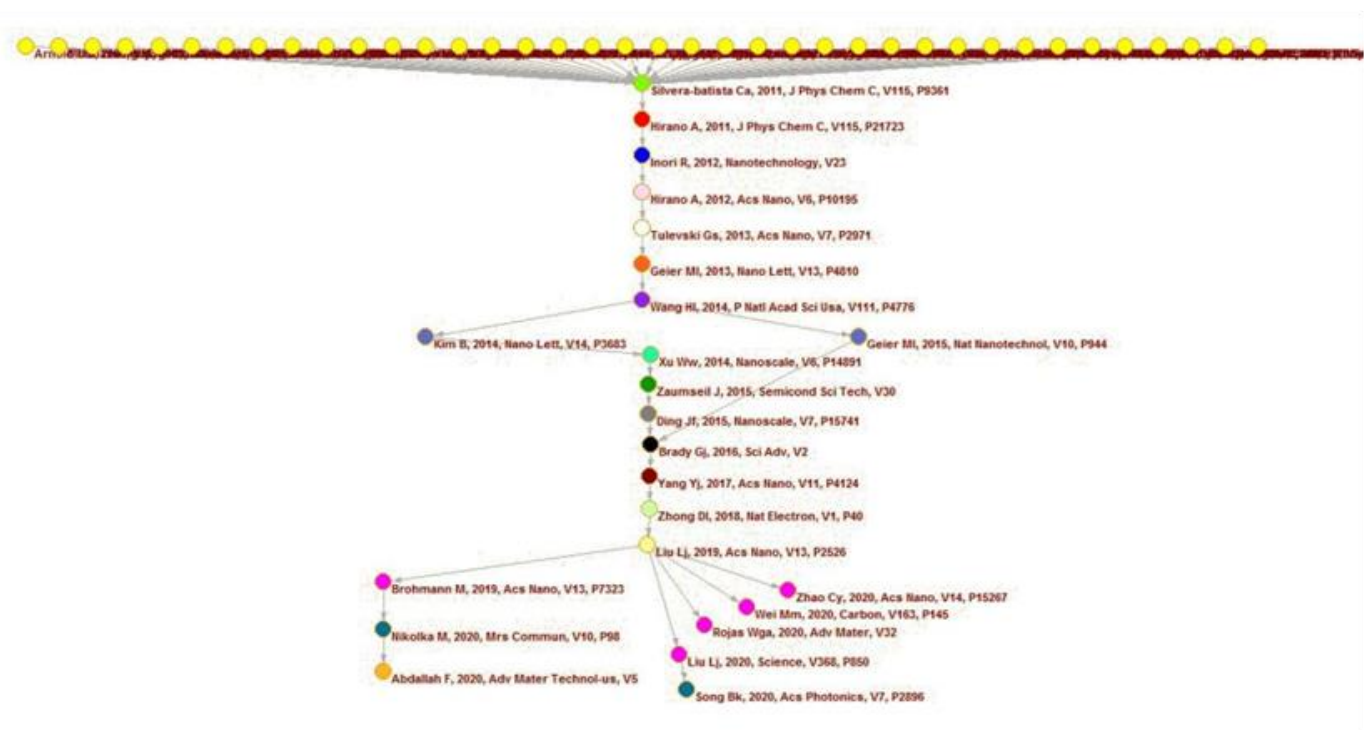

Figure 12. 2011 2020 Mainstream research paths in the field of the chip

First, from the structural point of view, the first stage formed two parts of noncrossing mainstream research paths; the second stage sources first converged at the first key node, formed three branches at the second key node, and then two of them converged into one path, and the third branch was divided into two paths, the first one merged into the path composed of the first two branches, and the other one formed a new path alone; the third stage shows a structure of concentration in the early stage and decentralization in the later stage. Second, from the viewpoint of scientific literature affiliation, the first stage of the mainstream research path only contains four countries, the United States, Germany, Japan and Spain, of which the United States has $66.5 \%$ of scientific papers, occupying the top of the list, which indicates that in the early research these countries are more influential in the field of the chip, and the main flow of knowledge also occurred in these four countries. In the second stage, Canada, France, Korea, China and India are new countries on the main path, but the dominant country is still the United States. In the third stage, the new countries in the main path are the United Kingdom and Egypt. Finally, in terms of content, the first phase paper exploring the electrical properties of metal-insulator-semiconductor capacitors by Mui, Liaw, Demirel, Strite, and Morkoc(1991) and the paper exploring a unique approach to characterize heterogeneous interfaces at the atomic scale and imaging inhomogeneities in semiconductors by Christen, Grundmann and Bimberg(1991) are the key nodes of the mainstream research path in this segment, and they cite a large amount of literature from the previous phase and two parts of the clear mainstream research path are formed from here. The first part focuses on organometallic vapor phase epitaxy and Raman spectroscopy for chip fabrication, while the other part focuses on the electrical properties of different material films and the interfacial properties of metal-insulator-semiconductor (MIS) capacitors, etc. The Research on materials that form nanoscale magnetic field regions by Theodoropoulou et al. (2001) is the first key node of the second phase, followed by a study related to ferromagnetism of chip fabrication materials (Overberg et al., 2001). Then the main path shows a trend of dispersion on both sides, which focuses on the ferromagnetism of thin-film materials, antiferromagnetic coupling, and doping and annealing techniques in the chip fabrication process. The third phase focuses on the research related to the new generation of materials carbon-based nanotubes as well as thin film nanotubes.

\section{Conclusion and Discussion}

This study depicts the dynamic landscape of scientific research in the field of the chip, providing a comprehensive landscape of the current scale of output and growing trend in the field of the chip at three levels: countries/regions, organizations, and scientific papers, respectively, answering the key questions of where scientific knowledge in the field of the chip is mainly located, which organizations are the major players in chip research, how their innovation distribution changes over time, and what are the mainstream research paths in the field, aiming to provide a detailed understanding of how to carry out innovation activities in the field for researchers, policymakers and research funders.

Specifically, this study finds a significant trend of increasing scientific paper output in the field of the chip in general. First, in terms of geographical location, about $94 \%$ of the world's scientific knowledge in the chip area 
is concentrated in the TOP 10 countries/regions, with the highest chip research intensity in the U.S., China and Japan, followed by Germany, South Korea and Taiwan. This study then tracks the longitudinal evolution of the top 10 countries/regions and finds that the scientific research output in the field of chip in the U.S. is declining year by year, while China has achieved a steady and rapid growth and surpassed the U.S. as the world's top output country in 2017, Japan has experienced a development phase of rising and then declining, and all other countries/regions have a relatively flat output curve. The University of California, the Chinese Academy of Sciences, and National Chiao Tung University are the top three high-producing institutions in the field of chip research. Among the TOP20 high-producing institutions, institutions affiliated with the U.S. occupy 30\% of the first place and Taiwan occupies $20 \%$ of the second place, which shows the importance and excellent results of chip research in Taiwan. And the proportion of universities in the TOP20 institutions is as high as $70 \%$, followed by research institutes and enterprises, which shows that the basic research in the chip field is carried out in universities.

Second, in terms of cooperation, this study divides the entire study period into three-time windows to analyze the dynamic landscape of cooperation and finds that both national/regional cooperation networks and institutional cooperation networks show a significant trend of expansion. Among the national/regional scientific cooperation networks, a core group of cooperation with Germany, the United States, and the United Kingdom has formed in each of the three-time periods. In addition to the United States and Germany, some neighboring countries/regions such as Japan, South Korea, and Taiwan, have been cooperating most closely with China. The US and Germany have been at the core of the network, indicating that they have always attached importance to international cooperative research, while China was initially located at the edge of the network, and only occupied the core of the network in the third phase, indicating that China is gradually expanding the degree of foreign cooperation. In the first two phases of the institutional cooperation network, the institution with the highest intensity of cooperation has been the University of California, and in the third phase, the Chinese Academy of Sciences overtook the University of California as the organization with the highest intensity of cooperation in the world, and the number of Chinese institutions in the cooperation network gradually increased, with the proportion of institutions affiliated with China reaching $26 \%$ in the third phase. In addition, the institutional collaboration network has produced distinct regional clusters, i.e., innovators tend to collaborate more with other institutions that are geographically close to them, and the pattern of university-scientific research institutes collaboration has become more pronounced.

Finally, from the analysis of the main path in the field of chip research, it can be seen that the four countries with more achievements in the field of chips in the early stage are the United States, Germany, Japan and Spain. The main flow of knowledge in the field of the chip occurs in these countries, and the later entrants on the main path are China, France and Canada, etc. China has performed well in the third stage, occupying one-third of the number of articles on the main path, which is the same as the United States. In terms of content, the research topics on the main path in the chip field are focused on chip fabrication, including electrical properties of metal-insulator-semiconductor capacitors, materials of thin films, types of chip fabrication materials and their ferromagnetism, as well as doping and annealing techniques in the chip fabrication process.

The results of this study can be used by countries/regions as well as organizations to understand the development of their own and competitors' chip research fields, which can be used as one of the considerations for research decisions, and also as a reference for research funders. In addition, this study finds that chip research is concentrated in a very small number of countries/regions and organizations, which may lead to information and power asymmetry between established innovators and new entrants in the field, and new policies should promote synergistic development in the future. The limitations of this study are the following three points: first, this study constructs the search formula from the manufacturing process of chips based on the reference of existing studies, and its accuracy needs to be improved; second, there are many kinds of chips, and this study only depicts the overall outline of the innovation dynamics in this field, and future studies can subdivide chips and analyze the innovation status of their specific subfields; third, this study only focuses on the chip field scientific development, and future research can start to study its technological and industrial aspects, which can help to comprehensively understand the comprehensive innovation value of a country/region and institution.

\section{Acknowledgement}

Supported by National Natural Science Foundation of China, "Research on the Structure, Evolution and Performance Influencing Mechanism of Multi-level Innovation Network in Frontier Science and Technology Areas for Innovation Chain" (grant number 72174112); The National Natural Science Foundation of China, "Mechanism, Evolution and Measurement of Technology Convergence Emergence" (grant number 71672103). 


\section{References}

Boly, V., Morel, L., \& Camargo, M. (2014). Evaluating innovative processes in french firms: Methodological proposition for firm innovation capacity evaluation. Research Policy, 43(3), 608-622. https://doi.org/10.1016/j.respol.2013.09.005

Cao, X., Zhang W., \& Zhang Y. (2017). Measurement and evaluation of independent technological innovation capability of strategic emerging industries. Journal of Central South Universi ty (Social Science Edition), 1, 101-109. https://doi.org/10.3969/j.issn.1672-3104.2017.01.014

Chen, K., \& Liu, D. (2020). Research on integrated circuit test technology. Electronic Compone-nts and Information Technology, 8, 12-14.

Christen, J., Grundmann, M., \& Bimberg, D. (1991). Scanning cathodoluminescence microscopy: A unique approach to atomic-scale characterization of heterointerfaces and i-maging of $\mathrm{s}$-emiconductor inhomogeneities. Journal of Vacuum Science \& Technology B: Microelectro-nics and Nanometer Structures Processing, Measurement, and Phenomena, 9(4), 2358-2368. https://doi.org/10.1116/1.585704

Cui, J., \& Zhang, G. J. (2017). Technology Development of Integrated Circuit Chips through China's Patent Status. (eds.) Proceedings of the Thirty-first China (Tianjin) 2017' Academic Conference on IT, Networking, Information Technology, Electronics, and Instru-mentation I-nnovation (pp. 84-87). Tianjin Electronics Society, Tianjin Instrumentation S-ociety.

Frame, J. D. (1977). Mainstream research in Latin America and the Caribbean. Interciencia, 2(3), 143-148.

Guan, J., \& Liu, N. (2014). Measuring scientific research in emerging nanoenergy field. Journ-al of Nanoparticle Research, 16(4), 1-15. https://doi.org/10.1007/s11051-014-2356-8

Hummon, N. P., \& Dereian, P. (1989). Connectivity in a citation network: The development of DNA theory. Social networks, 11(1), 39-63. https://doi.org/10.1016/0378-8733(89)90017-8

Iino, T., Inoue, H., Saito, Y. U., \& Todo, Y. (2021). How does the global network of researc-h collaboration affect the quality of innovation? The Japanese Economic Review, 72, 5-48. https://doi.org/10.1007/s42973-020-00042-y

Lee, J., Swain, B., Gu, B. W., Lee, C. G., \& Yoon, J. H. (2021). Value extraction from sem-iconductor industry tantalum scrap through understanding the thermodynamics and chemistr-y. International Journal of Refractory Metals and Hard Materials, 100, 105641. https://doi.org/10.1016/j.jirmhm.2021.105641

Li, L. T. (2019). Research Report on the Progress of Artificial Intelligence Chip Technology an-d Industrial Development. Xiamen Science and Technology, 1, 1-9.

Li, M., Chen, F. L., Luo, X. J., Yang, L. J., \& Zhang, J. (2021). Fundamental principles, key t-echnologies and research progress of atomic chips. Journal of Physics, 2, 56-75.

Liu, J. S., \& Lu, L. Y. (2012). An integrated approach for main path analysis: Development o-f the Hirsch index as an example. Journal of the American Society for Information Scien-ce and Technology, 63(3), 528-542. https://doi.org/10.1002/asi.21692

Liu, M. Y. (2021). Research on integrated circuit design methods and IP design techniques. El-ectronic Testing, 4, 12-14. https://doi.org/10.3969/j.issn.1000-8519.2021.04.003.

Liu, Y. H., Shi, A. J., Li, Z. W. \& He, X. J. (2020). Patent cooperation network structure an-d link prediction in Guangdong-Hong Kong-Macau-Greater Bay Area - taking chip field as an example. China Market, 35, $32-35$.

Liu, Y., Yan, Z., Cheng, Y., \& Ye, X. (2018). Exploring the technological collaboration chara-cteristics of the global integrated circuit manufacturing industry. Sustainability, $10(1), \quad 196$. https://doi.org/10.3390/su10010196

Manz, A., \& Eijkel, J. C. (2001). Miniaturization and chip technology. What can we expect? Pure and Applied Chemistry, 73(10), 1555-1561. https://doi.org/10.1351/pac200173101555

Mao, J. Q., Sun, J. L. \& Liu, N. (2016). Scientific capability measurement of energy storage tec-hnology in China and international comparison. Science Research Management, 12, 19-27.

Mui, D. S. L., Liaw, H., Demirel, A. L., Strite, S., \& Morkoc, H. (1991). Electrical character-istics of $\mathrm{Si} 3 \mathrm{~N} 4 / \mathrm{Si} / \mathrm{GaAs}$ metal-insulator-semiconductor capacitor. Applied physics letters, 59(22), 2847-2849. https://doi.org/10.1063/1.105853 
Overberg, M. E., Abernathy, C. R., Pearton, S. J., Theodoropoulou, N. A., McCarthy, K. T., \& Hebard, A. F. (2001). Indication of ferromagnetism in molecular-beam-epitaxy-derived N-type GaMnN. Applied Physics Letters, 79(9), 1312-1314. https://doi.org/10.1063/1.1397763

Rivette, K. G., \& Kline, D. (2000). Rembrandts in the attic: Unlocking the hidden value of patents. Harvard Business Press.

Smith, T., Sonnenfeld, D. A., \& Pellow, D. N. (Eds.). (2006). Challenging the chip: Labor rig-hts and environmental justice in the global electronics industry. Temple University Press.

Theodoropoulou, N., Lee, K. P., Overberg, M. E., Chu, S. N. G., Hebard, A. F., Abernathy, C. R., ... Wilson, R. G. (2001). Nanoscale magnetic regions formed in GaN implanted wi-th Mn. Journal of nanoscience and nanotechnology, 1(1), 101-106. https://doi.org/10.1166/jnn.2001.004

Verspagen, B. (2007). Mapping technological trajectories as patent citation networks: A study o-n the history of fuel cell research. Advances in complex systems, 10(01), 93-115. https://doi.org/10.1142/S0219525907000945

Vo-Dinh, T., \& Cullum, B. (2000). Biosensors and biochips: advances in biological and medic-al diagnostics. Fresenius' journal of analytical chemistry, 366(6), 540-551. https://d-oi.org/10.1007/s002160051549

Wang, H. Q., \& Hou, Y. (2017). Research on the evaluation of technological innovation capab-ility of Chinese high-tech industries. China Science and Technology Forum, 3, 58-63.

Wang, L. N., Tang, C., \& Xu, J. (2020). Analysis of future chip technology development trends. World Science and Technology Research and Development, 42(01), 47-56.

Wang, Y. J. (2020). What is the "stuck neck" problem stuck in. Science and Society, 10(04), 9-11.

Xi, J. P. (2020). Speech at the Symposium on Scientists. People's Daily. https://doi.org/10.3969/j.issn.1003-1871.2020.19.002

Yamada, K., Shibata, H., Suzuki, K., \& Citterio, D. (2017). Toward practical application of paper-based microflu idics for medical diagnostics: state-of-the-art and challenges. Lab on a C-hip, 17(7), 1206-1249. https://doi.org/10.1039/C6LC01577H

Zou, C. (2020). Microwave adaptive optical imaging processing technology based on AI chip. S-cience and Technology Innovation, 33, 26-28.

\section{Copyrights}

Copyright for this article is retained by the author(s), with first publication rights granted to the journal.

This is an open-access article distributed under the terms and conditions of the Creative Commons Attribution license (http://creativecommons.org/licenses/by/4.0/). 